\title{
Option Pricing under Delay Geometric Brownian Motion with Regime Switching
}

\author{
Tianyao Fang, Liangjian Hu, Yun Xin \\ Department of Applied Mathematics, Donghua University, Shanghai, China
}

Email address:

Tyfang995@gmail.com (Tianyao Fang),Ljhu@dhu.edu.cn (Liangjian Hu), Xinyun317@163.com (Yun Xin)

\section{To cite this article:}

Tianyao Fang, Liangjian Hu, Yun Xin. Option Pricing under Delay Geometric Brownian Motion with Regime Switching. Science Journal of Applied Mathematics and Statistics. Vol. 4, No. 6, 2016, pp. 263-268. doi: 10.11648/j.sjams.20160406.13

Received: September 25, 2016; Accepted: October 13, 2016; Published: October 18, 2016

\begin{abstract}
We investigate the option pricing problem when the price dynamics of the underlying risky assets are driven by delay geometric Brownian motions with regime switching. That is, the market interest rate, the appreciation rate and the volatility of the risky assets depend on the past stock prices and the unobservable states of the economy which are modulated by a continuous-time Markov chain. The market described by the model is incomplete, the martingale measure is not unique and the Esscher transform is employed to determine an equivalent martingale measure. We proved the model has a unique positive solution and the price of the contingent claims under the model can be computable numerically if not analytically.
\end{abstract}

Keywords: Option Pricing, Regime Switching, Esscher Transform, Itô Formula, Euler-Maruyama

\section{Introduction}

In the modern financial economics, options form a very important and useful class of financial securities. There is an important problem from both theoretical and practical perspectives is that how to determine options value. In the path- breaking works of Black, Scholes [1] and Merton [16] assume that the price dynamics of the underlying risky assets are driven by a geometric Brownian motion (GBM). The option pricing formula does not rely on the actual appreciation rate of the underlying risky asset, which is replaced by the risk-free rate of interest.

Despite its compact form and popularity, the fitness of the GBM model has been questioned on the basis of the assumption of constant volatility, as empirical evidence shows that volatility actually depends on time in a way that is not predictable. Moreover, the need for better ways of understanding the behavior of many natural processes has motivated the development of dynamic models of these processes that take into consideration in the effect of past events on the current and future states of the system. This view is especially appropriate in the research of financial variables, since predictions about their evolution take strongly into account the knowledge of their past. For example, Arriojas, Hu et al. [2] develop an explicit formula for pricing European options when assume the stock prices satisfy a stochastic differential delay equation. The model maintains the no-arbitrage property and the completeness of the market too. Yan and Zhang [3] priced the European options when the underlying assets price follows the stochastic functional differential equation with finite delay driven by a G-Brownian motion. Mao and Sabanis [4] propose a model driven by a delay geometric Brownian motion and introduce an Euler-Maruyama numerical scheme for their model which shows that this method approximates options price very well.

In recent years, regime switching models become more and more important in modern financial economics. The origin of econometric applications of regime switching can track back to the early work of Hamilton [5] in which a class of discrete-time Markov switching autoregressive time series models is proposed. These models provide a more realistic way to describe the asset price dynamics for option pricing. They can incorporate the effect of structural changes in macro-economic conditions and business cycles on option price. Nowadays, the applications of Markov regime switching models can be found in various important fields in financial economics. Some of these applications include Elliott, Chan and Siu [6, 7, 8] consider the option pricing problem when the risky underlying assets are driven 
by geometric Brownian motions with regime switching. Ratanov [9] studies incomplete market models based on jump-diffusion processes with parameters that are switched at random times and obtain explicit formula for the option prices in the two-state hidden Markov process. Ma and Zhou [10] investigate moving mesh implicit finite differen-ce methods for pricing Asian options with regime switching which follows a system of partial differential equations with moving boundaries. Fan, Shen et al. [14] discuss a Markov chain approximation method to price European options, American options and barrier options in a Markovian regime-switching environment. Jin and Qian [15] introduce a numerical method to price the European lookback floating strike put options where the underlying asset price is modeled by a generalized regime-switching jump diffusion process. However there is little amount of work on the option pricing when consider the underlying assets prices are affected by the past stock prices and the unobservable states of the economy.

In this paper, we investigate the option pricing problem when the price dynamics of the underlying risky assets are governed by a delay geometric Brownian motion with regime switching. In particular, the market interest rate, the appreciation rate and the volatility of the risky assets depend on the past stock prices and the unobservable states of the economy which are modulated by an continuous-time Markov chain. More specifically, one may interpret the states of the markov chain as proxies of observable macro-economic indicators, such as gross domestic product (GDP) and retail price index (RPI), or different stages of business cycles. The market described by the model is incomplete in general, hence the martingale measure is not unique. We adopt a regime switching Esscher transform to determine an equivalent martingale measure. Furthermore, we prove the model has a unique positive solution and the price of contingent claims under the model can be computable numerically if not analytically.

\section{The Delay Geometric Brownian Motion with Regime Switching}

Throughout this paper, unless otherwise specified, we let $\left(\Omega, \mathcal{F},\left\{\mathcal{F}_{t}\right\}_{t>0}, \mathbb{P}\right)$ be a complete probability space with a filtration $\left\{\mathcal{F}_{t}\right\}_{t \geq 0}$ satisfying the usual conditions (i.e. it is increasing and right continuous while $\mathcal{F}_{0}$ contains all $\mathbb{P}$-null sets). Let $W(t), t \geq 0$ be a scalar Brownian motion defined on the probability space. If $x, y$ are real numbers, the $x \vee y$ denotes the maximum of $x$ and $y$, and $x \wedge y$ denotes the minimum of $x$ and $y$. Let $\tau>0$ and $C\left([-\tau, 0], \mathbb{R}_{+}\right)$ denotes the family of continuous function $\xi$ : from $[-\tau, 0]$ to $R_{+}$with the norm $\|\xi\|=\sup _{-\tau \leq u \leq 0} \xi(u)$.

Let $\{X(t), t \in[0, T]\}$ be a right-continuous Markov chain on the probability space taking values in a finite state space $\mathcal{N}=\{1,2, \ldots, N\}$ with generator $\Gamma=\left(\gamma_{i j}\right)_{N \times N}$ given by:

$$
P\{X(t+\Delta)=j \mid X(t)=i\}= \begin{cases}\gamma_{i j} \Delta+o(\Delta) & \text { if } i \neq j \\ 1+\gamma_{i j} \Delta+o(\Delta) & \text { if } i=j\end{cases}
$$

where $\Delta>0$. Hence $\gamma_{i j}>0$ is transition rate from $i$ to $j$ if $i \neq j$ while:

$$
\gamma_{i i}=-\sum_{i \neq j} \gamma_{i j}
$$

We assume that the Markov chain $X(\cdot)$ is independent of the Brownian motion $W(\cdot)$. It is well known that almost every sample path of $X(t)$ is right continuous step function and $X(t)$ is ergodic Markov chain. Moreover, $\phi$ denotes the empty set and we set inf $\phi=\infty$. For a set A, its indication function is denoted by $I_{A}$.

Consider the asset price process $S(t)$ be driven by the delay geometric Brownian motion with regime switching:

$$
\begin{aligned}
d S(t) & =\mu(S(t-\tau), X(t)) S(t) d t \\
& +\sigma(S(t-\tau), X(t)) S(t) d W(t)
\end{aligned}
$$

on $t \geq 0$, with initial data $S(0)=\xi(u) \in C\left([-\tau, 0] ; \mathbb{R}_{+}\right)$. Here $\bar{\tau}$ is a positive constant, $r(X(t))>0$ is the risk-free interest rate and $W(t)$ is a scalar Brownian motion. While the appreciation rate $\mu(S(t-\tau), X(t))$ and the volatility $\sigma(S(t-\tau), X(t))$ are in $C\left(\mathbb{R}_{+} ; \mathbb{R}_{+}\right)$.

\section{Equivalent Martingale Measure}

Since the market described by the model (1) is incomplete, there are more than one equivalent martingale measures. Based on the risk-neutral pricing theory, the option valuation is equal to the conditional expectation of the discounted maturity function under risk-neutral measure. So having a reasonable equivalent martingale measure is crucial to the asset pricing in incomplete market. Following from Elliot et al. [6], we employ the regime switching Esscher transform to determine an equivalent martingale measure. Let

$$
\begin{aligned}
Y_{t} & =\ln \frac{S_{t}}{S_{0}}=\int_{0}^{t}[\mu(S(u-\tau), X(u)) \\
& \left.-\frac{1}{2} \sigma^{2}(S(u-\tau), X(u))\right] d u \\
& +\int_{0}^{t} \sigma(S(u-\tau), X(u)) d W(u) .
\end{aligned}
$$

Suppose $\left\{\mathcal{F}^{Y}\right\}_{t \in[0, T]}, \quad\left\{\mathcal{F}^{X}\right\}_{t \in[0, T]}$ denote the $\mathbb{P}$ -augments of the natural filtrations generated by $\left\{Y_{t}, t \in[0, T]\right\}, \quad\left\{X_{t}, t \in[0, T]\right\}$ respectively. For each $t \in[0, T]$, set $\mathcal{H}_{t}=\mathcal{F}_{t}^{Y} \vee \mathcal{F}_{t}^{X}$. Then we can define a regime switching Esscher transform $\mathbb{Q} \sim \mathbb{P}$ on $\mathcal{H}_{T}$ with respect to a family of parameters $\theta_{t}$ by:

$$
\frac{d \mathbb{Q}}{d \mathbb{P}}=\frac{\exp \left(\int_{0}^{T} \theta_{t} d Y_{t}\right)}{\mathbb{E}\left[\exp \left(\int_{0}^{T} \theta_{t} d Y_{t}\right) \mid \mathcal{F}_{T}^{X}\right]},
$$

while, $\mathbb{E}[\cdot]$ denote the expectation under $\mathbb{P}$.

Note that given $\mathcal{F}_{T}^{X}, \int_{0}^{T} \theta_{t} d Y_{t}$ follows a normal 
distribution with mean:

$$
\int_{0}^{T} \theta_{t}\left[\mu(S(t-\tau), X(t))-\frac{1}{2} \sigma^{2}(S(t-\tau), X(t))\right] d t
$$

and variance $\int_{0}^{T} \theta_{t}^{2} \sigma^{2}(S(t-\tau), X(t)) d t$ under $\mathbb{P}$.

So the Radon-Nikodym derivative can be written as:

$$
\begin{aligned}
\frac{d \mathbb{Q}}{d \mathbb{P}} & =\exp \left\{\int_{0}^{T} \theta_{t} \sigma(S(t-\tau), X(t)) d t\right. \\
& \left.-\frac{1}{2} \theta_{t}^{2} \sigma^{2}(S(t-\tau), X(t)) d t\right\}
\end{aligned}
$$

Moreover, we assume that:

$$
\mathbb{E}\left[\exp \left(\frac{1}{2} \int_{0}^{T} \theta_{t}^{2} \sigma^{2}(S(t-\tau), X(t)) d t\right)\right]<\infty .
$$

By the fundamental theorem of asset pricing, the absence of arbitrage is "essentially" equivalent to the existence of an equivalent martingale measure under which the discounted stock price process is a martingale. As in Elliot et al. [6], due to the presence of the uncertainly generated by the Markov process $X$, the martingale condition in our case is given by $\tilde{S}(t)=\tilde{\mathbb{E}}\left[\tilde{S}(T) \mid \mathcal{H}_{t}\right]$ which is defined with respect to the enlarged filtration $\mathcal{H}_{t}$ under the probability $\mathbb{Q}$. Here $\tilde{\mathbb{E}}[\cdot]$ denotes the expectation under $\mathbb{Q}$. Then, by applying Bayes rule, we can obtain that the martingale condition holds if and only if :

$$
\theta_{t}=\frac{r(X(t))-\mu(S(t-\tau), X(t))}{\sigma^{2}(S(t-\tau), X(t))},
$$

for all $t \in[0, T]$.

According to the martingale condition, the Radon-Nikodym derivative of $\mathbb{Q}$ is given by:

$$
\begin{aligned}
\frac{d \mathbb{Q}}{d \mathbb{P}} & =\exp \left\{\int_{0}^{T}\left[\frac{r(X(t))-\mu(S(t-\tau), X(t))}{\theta_{t}}\right] d W(t)\right. \\
& \left.-\frac{1}{2} \int_{0}^{T}\left[\frac{r(X(t))-\mu(S(t-\tau), X(t))}{\theta_{t}}\right]^{2} d t\right\}
\end{aligned}
$$

By Girsanov's theorem, we can obtain the following result, given $\mathcal{F}_{T}^{X}$

$$
\tilde{W}_{t}=W_{t}+\int_{0}^{t} \frac{r(X(u))-\mu(S(u-\tau), X(u))}{\sigma(S(u-\tau), X(u))} d u
$$

is a standard $\mathbb{Q}$-Brownian motion. Because the Markov chain $X(\cdot)$ is independent of the Brownian motion $W(\cdot)$, the formula of Markov chain $X(\cdot)$ will not be changed under a regime switching Esscher transform $\mathbb{P} \sim \mathbb{Q}$.

Furthermore, the stock price process $S(t)$ satisfying the following model:

$$
\begin{aligned}
d S(t) & =r(X(t)) S(t) d t \\
& +\sigma(S(t-\tau), X(t)) S(t) d \tilde{W}(t)
\end{aligned}
$$

As a result, the discount asset price process is a martingale and the model is arbitrage free. The risk-neutral measure $\mathbb{Q}$ guarantees that the market is complete and appropriate hedging strategies can be obtained.

Again for the reason of notational simplicity, although we will work with the "risk-neutral" probability space $\left(\Omega, \mathcal{F},\left\{\mathcal{F}_{t}\right\}_{t \geq 0}, \mathbb{Q}\right)$, we will avoid the use of the " $\sim$ " notation for all relevant calculations in the next sections:

$$
\begin{aligned}
d S(t) & =r(X(t)) S(t) d t \\
& +\sigma(S(t-\tau), X(t)) S(t) d W(t)
\end{aligned}
$$

\section{Existence and Uniqueness of a Positive Solution}

The model (3) describes the option price and its volatility in the financial market. It is therefore essential to prove that the solution of model (3) is non-negative with probability 1 [11]. We impose the following assumption.

Assumption 4.1 When the state of markov chain $X(t)$ is $i$, the volatility function $\sigma$ is bounded by a positive constant $K_{i}$, namely,

$$
|\sigma(S(t-\tau), i)| \leq K_{i} \quad \forall i \in \mathcal{N},
$$

where $K=\max _{i \in \mathcal{N}} K_{i}$.

According to Assumption 4.1, we are not surprise to have both the drift and diffusion coefficients of model (3) are local Lipschitz continuous and satisfy the linear growth condition.

Theorem 4.2 Given any initial value $S(0)=\xi(u)>0$, there exist a unique global positive solution $S(t)$ to model (3) on $[0, T]$, namely,

$$
P\left\{S(t) \in R_{+}, \forall t \in[0, T]\right\}=1 .
$$

Proof. Clearly, the drift and diffusion coefficients of model (3) obey the local Lipschitz continuous. Hence, there exist a unique maximal solution $S(t)$ on $\left[-\tau, \rho_{e}\right]$, where $\rho_{e}$ is the explosion time [12]. To prove our theorem we need to show that $\rho_{e}=T$ a.s..

For each sufficiently large integer $k_{0}$, satisfying:

$$
\frac{1}{k_{0}} \leq S(0)=\|\xi\|=\sup _{-\tau \leq u \leq 0}|\xi(u)| \leq k_{0},
$$

such that for any $k \geq k_{0}$, define the stopping time:

$$
\rho_{k}=T \wedge \inf \{t \in[0, T]:|S(t)| \geq k\} .
$$

Obviously, $\rho_{k}$ is increasing as $k \rightarrow \infty$. Set $\rho_{\infty}=\lim _{k \rightarrow \infty} \rho_{k}$, hence $\rho_{\infty} \leq \rho_{e}$ a.s. If we can prove $\rho_{\infty}=T$ a.s., then $\rho_{e}=T$ a.s., and $S(t) \geq 0, \forall t \in[0, T]$ a.s.. In other words, to complete the proof we need to show: $\rho_{\infty}=T$ a.s.

Next we prove it by contradiction, namely we can find a sufficiently large $k_{1}$ for $P\left\{\rho_{k} \leq T\right\}>\varepsilon, \forall k \geq k_{1}$.

Define the function $V: \mathbb{R}_{+} \times \mathbb{R}_{+} \times[0, T] \times \mathcal{N} \rightarrow \mathbb{R}_{+}$, by

$V(S(t), S(t-\tau), t, i)=U(S(t))=(1+|S(t)|)^{\frac{p}{2}}, \quad p \geq 2$

and we compute the diffusion operator: 


$$
\begin{aligned}
& L V(S(t), S(t-\tau), t, i) \\
& =V_{t}(S(t), t, i)+V_{S}(S(t), t, i) r(i) S(t) \\
& +\frac{1}{2} \operatorname{trace}\left[(\sigma(S(t-\tau), i) S(t))^{T} V_{S S}(S(t), t, i)\right. \\
& \cdot \sigma(S(t-\tau), i) S(t)]+\sum_{j=1}^{N} \gamma_{i j} V(S(t), t, j) \\
& \leq p\left(1+|S(t)|^{2}\right)^{\frac{p}{2}-1} \bar{H}_{K}\left(1+|S(t)|^{2}+|S(t-\tau)|^{2}\right) \\
& +\frac{p}{2}\left(1+|S(t)|^{2}\right)^{\frac{p}{2}-1} \bar{H}_{K}\left(1+|S(t)|^{2}+|S(t-\tau)|^{2}\right) \\
& +\frac{p(p-2)}{2}\left(1+|S(t)|^{2}\right)^{\frac{p}{2}-2}|S(t)|^{2} \bar{H}_{K} \\
& \cdot\left(1+|S(t)|^{2}+|S(t-\tau)|^{2}\right) \\
& \leq \frac{3}{2} \bar{H}_{K} p\left(1+|S(t)|^{2}\right)^{\frac{p}{2}} \frac{1+|S(t)|^{2}+|S(t-\tau)|^{2}}{1+|S(t)|^{2}} \\
& +\frac{\bar{H}_{K} p(p-2)}{2}\left(1+|S(t)|^{2}\right)^{\frac{p}{2}-2}\left(1+|S(t)|^{2}\right) \\
& \cdot\left(1+|S(t)|^{2}+|S(t-\tau)|^{2}\right) \\
& =\frac{\bar{H}_{K} p(p+1)}{2}\left[\left(1+|S(t)|^{2}\right)^{\frac{p}{2}}\right. \\
& \left.+\left(1+|S(t)|^{2}\right)^{\frac{p}{2}-1}|S(t-\tau)|^{2}\right] .
\end{aligned}
$$

By the elementary inequality:

$$
a^{c} b^{1-c} \leq a c+b(1-c), \quad \forall a, b \geq 0, c \in[0,1],
$$

we have

$$
\begin{aligned}
& \left(1+|S(t)|^{2}\right)^{\frac{p}{2}-1}|S(t-\tau)|^{2} \\
& \leq \frac{\frac{p}{2}-1}{\frac{p}{2}}\left(1+|S(t)|^{2}\right)^{\frac{p}{2}}+\frac{1}{\frac{p}{2}}\left(1+|S(t-\tau)|^{2}\right)^{\frac{p}{2}} \\
& =\left(1-\frac{2}{p}\right)\left(1+|S(t)|^{2}\right)^{\frac{p}{2}}+\frac{2}{p}\left(1+|S(t-\tau)|^{2}\right)^{\frac{p}{2}} .
\end{aligned}
$$

Hence,

$$
\begin{aligned}
& L V(S(t), S(t-\tau), t, i) \\
& \leq \frac{\bar{H}_{K} p(p+1)}{2}\left[U(S(t))+\left(1-\frac{2}{p}\right) U(S(t))\right. \\
& \left.+\frac{2}{p} U(S(t-\tau))\right] \\
& \leq \bar{H}_{K} p(p+1)[U(S(t))+U(S(t-\tau))] .
\end{aligned}
$$

Then using the generalized Itô formula:

$$
\begin{aligned}
& \mathbb{E} U\left(S\left(t \wedge \rho_{k}\right)\right) \\
& =\mathbb{E} V\left(S\left(t \wedge \rho_{k}\right), t \wedge \rho_{k}, X\left(t \wedge \rho_{k}\right)\right) \\
& =\mathbb{E} V(S(0), 0, X(0)) \\
& +\mathbb{E} \int_{0}^{t \wedge \rho_{k}} L V(S(u), S(u-\tau), u, X(u)) d u \\
& \leq \alpha_{1}+\bar{H}_{K} p(p+1) \\
& \cdot \mathbb{E} \int_{0}^{t \wedge \rho_{k}}[U(S(u))+U(S(u-\tau))] d u \\
& \leq \alpha_{1}+2 \bar{H}_{K} p(p+1) \int_{0}^{t}\left[\sup _{-\tau<u<v} \mathbb{E} U\left(S\left(u \wedge \rho_{k}\right)\right)\right] d v
\end{aligned}
$$

where $\alpha_{1}=\mathbb{E} V(S(0), 0, X(0))$. Observe the right-handside term is increasing in $\mathrm{t}$, we must have:

$$
\begin{aligned}
& \sup _{0 \leq u \leq t} \mathbb{E} U\left(S\left(u \wedge \rho_{k}\right)\right) \\
& \leq \alpha_{1}+2 \bar{H}_{K} p(p+1) \int_{0}^{t}\left[\sup _{-\tau \leq u \leq v} \mathbb{E} U\left(S\left(u \wedge \rho_{k}\right)\right)\right] d v .
\end{aligned}
$$

So, we can get:

$$
\begin{aligned}
& \sup _{-\tau \leq u \leq t} \mathbb{E} U\left(S\left(u \wedge \rho_{k}\right)\right) \\
& \leq \sup _{-\tau \leq u \leq 0} \mathbb{E} U(\xi(u))+\sup _{0 \leq u \leq t} \mathbb{E} U\left(S\left(u \wedge \rho_{k}\right)\right) \\
& \leq \alpha_{2}+2 \bar{H}_{K} p(p+1) \int_{0}^{t}\left[\sup _{-\tau \leq u \leq v} \mathbb{E} U\left(S\left(u \wedge \rho_{k}\right)\right)\right] d v
\end{aligned}
$$

where $\alpha_{2}=\sup _{-\tau \leq u \leq 0} \mathbb{E} U(\xi(u))+\alpha_{1}$. By using the Gronwall inequality implies:

$$
\sup _{-\tau \leq u \leq T} \mathbb{E} U\left(S\left(u \wedge \rho_{k}\right)\right) \leq \alpha_{2} e^{2 \bar{H}_{K} p(p+1) T} .
$$

In particular,

$$
\mathbb{E} U\left(S\left(T \wedge \rho_{k}\right)\right) \leq \alpha_{2} e^{2 \bar{H}_{K} p(p+1) T} .
$$

On the other hand, if we define

$$
h_{k}=\inf \{U(S(t)):|S(t)| \geq k\},
$$

then $h_{k} \rightarrow \infty$, as $k \rightarrow \infty$ and

$$
\begin{aligned}
\alpha_{2} e^{2 \bar{H}_{K} p(p+1) T} & \geq \mathbb{E}\left[I_{\left\{\rho_{k} \leq T\right\}} U\left(S\left(\rho_{k}\right)\right)\right] \\
& =\left\{\rho_{k} \leq T\right\} \\
& \geq \varepsilon h_{k} .
\end{aligned}
$$

Letting $\quad k \rightarrow \infty$, then $\infty \geq \alpha_{2} e^{2 \bar{H}_{K} p(p+1) T}=\infty$, obviously it yields a contradiction. So we have $\rho_{\infty}=T$ a.s. that is, $\rho_{e}=T$ a.s. and $P\left\{S(t) \in R_{+}, \forall t \in[0, T]\right\}=1$. We complete the proof.

\section{Numerical Solutions}

For the purpose of defining the Euler-Maruyama numerical solutions to the model (3), we first extend the definition the 
volatility function $\sigma$ from $R_{+}$to $R$ by letting $\sigma(x)=\sigma(0)$ for $x<0$. It can make the solutions are always positive and the volatility function satisfies local Lipschitz condition. Given a stepsize $0<\Delta<\tau$, set $t_{k}=k \Delta$. Let $[a / \Delta]$ defines the integer part of the real number $a / \Delta$. Compute the discrete approximation solution: $S(t) \approx s\left(t_{k}\right)$ by setting $S_{0}(t)=\xi(t)$ on $-\tau \leq t \leq 0, X_{0}^{\Delta}=i_{0} \quad[13]$ and forming:

$$
\begin{aligned}
S_{k+1} & =S_{k}+f\left(S_{k}, S_{[(k \Delta-\tau) / \Delta]}, X_{k}^{\Delta}\right) \Delta \\
& +g\left(S_{k}, S_{[(k \Delta-\tau) / \Delta]}, X_{k}^{\Delta}\right) \Delta W_{k},
\end{aligned}
$$

where $f(S(t), S(t-\tau), X(t))=r(X(t)) S(t)$,

$$
g(S(t), S(t-\tau), X(t))=\sigma(S(t-\tau), X(t)) S(t),
$$

and $\Delta W_{k}=W\left(t_{k+1}\right)-W\left(t_{k}\right)$. Let:

$$
\begin{aligned}
& \tilde{S}_{1}(t)=S_{k}, \quad \tilde{S}_{2}(t)=S_{[(k \Delta-\tau) / \Delta]}, \\
& \tilde{X}(t)=X_{k}^{\Delta}, \quad t \in\left[t_{k}, t_{k+1}\right)
\end{aligned}
$$

and define the continuous EM approximate solution:

$$
\begin{aligned}
S_{k} & =S_{0}+\int_{0}^{t} f\left(\tilde{S}_{1}(u), \tilde{S}_{2}(u), \tilde{X}(u)\right) d u \\
& +\int_{0}^{t} g\left(\tilde{S}_{1}(u), \tilde{S}_{2}(u), \tilde{X}(u)\right) d W(u) .
\end{aligned}
$$

Note that $S\left(t_{k}\right)=S_{k}$, for any $k=0,1, \ldots,[T / \Delta]$.

In the next section, we will discuss the strong convergence of the EM method while the coefficients $f$ and $g$ satisfy local Lipschitz continuous. Let us now present a lemma for further use.

Lemma 5.1 ([13] $p$ th moment bounded) For any $p>2$, there is a constant $L$, which is dependent on only $p, T, \xi$ but independent of $\Delta$, such that the exact solution and the EM approximate solution to the model (3) have the property that:

$$
\mathbb{E}\left[\sup _{0 \leq t \leq T}|S(t)|^{p}\right] \vee \mathbb{E}\left[\sup _{0 \leq t \leq T}|s(t)|^{p}\right] \leq L .
$$

Proof. see $[12,13]$.

Theorem 5.2 Under lemma 5.1 and the coefficients $f, g$ are local Lipschitz continuous, the EM approximate solution convergences to the exact solution of the model (3) in the sense that:

$$
\lim _{\Delta \rightarrow 0} \mathbb{E}\left[\sup _{0 \leq t \leq T}|S(t)-s(t)|^{2}\right]=0 .
$$

Proof. fix a $p>2$. By Lemma 5.1, there exist a positive constant $L$ independent of $\Delta$ such that:

$$
\mathbb{E}\left[\sup _{0 \leq t \leq T}|S(t)|^{p}\right] \vee \mathbb{E}\left[\sup _{0 \leq t \leq T}|s(t)|^{p}\right] \leq L .
$$

For sufficiently large integer $D$, define the stopping times

$$
\begin{aligned}
& \hat{\rho}_{D}=\inf \{t \in[0, T]:|S(t)| \geq D\}, \\
& \tilde{\rho}_{D}=\inf \{t \in[0, T]:|S(t)| \geq D\},
\end{aligned}
$$

and $\beta_{D}=\hat{\rho}_{D} \wedge \tilde{\rho}_{D}$. Set

$$
\zeta(t)=S(t)-s(t) .
$$

Using the Young inequality: for $m^{-1}+n^{-1}=1$ and $\forall a, b, \delta$, we can get:

$$
a b \leq \frac{\delta}{m} a^{m}+\frac{1}{n \delta^{n / m}} b^{n} .
$$

So for any $\delta>0$,

$$
\begin{aligned}
\mathbb{E}\left[\sup _{0 \leq t \leq T}|\zeta(t)|^{2}\right] & =\mathbb{E}\left[\sup _{0 \leq t \leq T}|\zeta(t)|^{2} I_{\left\{\hat{\rho}_{D}>T, \tilde{\rho}_{D}>T\right\}}\right] \\
& +\mathbb{E}\left[\sup _{0 \leq t \leq T}|\zeta(t)|^{2} I_{\left\{\hat{\rho}_{D} \leq T, \tilde{\rho}_{D} \leq T\right\}}\right] \\
& \leq \mathbb{E}\left[\sup _{0 \leq t \leq T}\left|\zeta\left(t \wedge \beta_{D}\right)\right|^{2} I_{\left\{\beta_{D}>T\right\}}\right] \\
& +\frac{2 \delta}{p} \mathbb{E}\left[\sup _{0 \leq t \leq T}|\zeta(t)|^{p}\right] \\
& +\frac{1-2 / p}{\delta^{2 /(p-2)}} P\left\{\hat{\rho}_{D} \leq T \text { or } \tilde{\rho}_{D} \leq T\right\} .
\end{aligned}
$$

According to (4),

$$
\begin{aligned}
P\left\{\hat{\rho}_{D} \leq T\right\} & =\mathbb{E}\left[\frac{\left|S\left(\hat{\rho}_{D}\right)\right|^{p}}{D^{p}} I_{\left\{\hat{\rho}_{D} \leq T\right\}}\right] \\
& \leq \frac{1}{D^{p}} \mathbb{E}\left[\sup _{0 \leq t \leq T}|S(t)|^{p}\right] \\
& \leq \frac{L}{D^{p}}
\end{aligned}
$$

A similar result can be derived for $\tilde{\rho}_{D}$, hence

$$
P\left\{\hat{\rho}_{D} \leq T \text { or } \tilde{\rho}_{D} \leq T\right\} \leq \frac{2 L}{D^{p}} .
$$

Again according to (4),

$\mathbb{E}\left[\sup _{0 \leq t \leq T}|\zeta(t)|^{p}\right] \leq 2^{p-1}\left(\mathbb{E}\left[\sup _{0 \leq t \leq T}|S(t)|^{p}\right]+\mathbb{E}\left[\sup _{0 \leq t \leq T}|s(t)|^{p}\right]\right)$

$$
\leq 2^{p} L
$$

In the similar way, we can show that

$$
\mathbb{E}\left[\sup _{0 \leq t \leq T}\left|S\left(t \wedge \beta_{D}\right)-s\left(t \wedge \beta_{D}\right)\right|^{2}\right] \leq R_{D} \Delta+o(\Delta)
$$

where $R_{D}$ is a constant independent of $\Delta$. Using these bounds gives

$$
\begin{aligned}
& \mathbb{E}\left[\sup _{0 \leq t \leq T}|\zeta(t)|^{2}\right] \\
& \leq \mathbb{E}\left[\sup _{0 \leq t \leq T}\left|S\left(t \wedge \beta_{D}\right)-s\left(t \wedge \beta_{D}\right)\right|^{2}\right] \\
& +\frac{2^{p+1} \delta L}{p}+\frac{2(p-2) L}{p \delta^{2 /(p-2)} D^{p}} \\
& \leq R_{D} \Delta+o(\Delta)+\frac{2^{p+1} \delta L}{p}+\frac{2(p-2) L}{p \delta^{2 /(p-2)} D^{p}} .
\end{aligned}
$$


Given any $\varepsilon>0$, choose $\Delta$ sufficiently small, $\delta, D$ sufficiently large, so that:

$$
R_{D} \Delta+o(\Delta)<\frac{\varepsilon}{3}, \frac{2^{p+1} \delta L}{p}<\frac{\varepsilon}{3}, \frac{2(p-2) L}{p \delta^{2 /(p-2)} D^{p}}<\frac{\varepsilon}{3},
$$

substituting this into (7),

$$
\mathbb{E}\left[\sup _{0 \leq t \leq T}|\zeta(t)|^{2}\right]<\varepsilon .
$$

We complete the proof.

According to the strong convergence properties mentioned above, we can have the expected pay-off under numerical method converges to the correct expected pay-off when $\Delta t \rightarrow 0$ for all kinds of options.

For example, assume that one buys a European call option at $t=0, K$ denote the exercise price at the expiry date $T$ and the underlying asset price is described by the model (3). Thus, the pay-off of the European call option at $t=0$ is:

$$
C=\mathbb{E}\left[e^{-\int_{0}^{T} r(X(t)) d t}(S(T)-K)^{+}\right]
$$

So we can obviously show that:

$$
\lim _{\Delta t \rightarrow 0}\left|\mathbb{E}(S(T)-K)^{+}-\mathbb{E}(s(T)-K)^{+}\right|=0 .
$$

Consequently, for a sufficiently small $\Delta t$, European call option price: $\mathbb{E}\left[e^{-\int_{0}^{T} r(X(t)) d t}(S(T)-K)^{+}\right]$have a numerical solution:

$$
\mathbb{E}\left[e^{-\int_{0}^{T} r(X(t)) d t}(s(T)-K)^{+}\right] .
$$

\section{Conclusion}

The proposed model (3) which is described by a SDDE with Markovian switching, is more suitable to modeling the real financial market. We use Esscher transform to determine an equivalent martingale measure in incomplete market. The model has a unique positive solution is proved and the price of the contingent claims under the model can be computable numerically if not analytically.

\section{Acknowledgements}

The authors would like to thank the National Natural Science Foundation of China (11471071) and the Natural Science Foundation of Shanghai (14ZR1401200) for their financial support.

\section{References}

[1] F. Black, M. Scholes, "The Pricing of Options and Corporate Liabilities," Journal of Political Economy, 1973, vol. 81, pp. 637-659.

[2] M. Arriojas, Y. Hu, S. Mohammed and G. Pap, "A Delayed Black and Scholes Formula," Stochastic Analysis and Applications, 2007, 25, pp. 471-492.

[3] L. Yan and Q. Zhan, "Successive Approximation of SFDEs with Finite Delay Driven by G-Brownian Motion," Abstract and Applied Analysis, 2013, 6, pp. 189-206.

[4] X. Mao and S. Sabanis, "Delay Geometric Brownian Motion in Financial Option Valuation," Journal of Probability and Stochastic Processes, 2013, 85(2), pp. 295-320.

[5] J. Hamilton, "A New Approach to the Economic Analysis of Nonstationary Time Series and the Business Cycle," Ecomometrica, 1989, 57, pp. 357-384.

[6] J. Elliott, L. Chan and T. Siu, "Option Pricing and Esscher Transform under Regime Switching," Annals of Finance, 2005, pp. 423-432.

[7] J. Elliott, L. Chan and T. Siu, "Option Valuation under a Regime-Switching Constant Elasticity of Variance Process," Applied Mathematics and Computation, 2013, 219(9), pp. 4434-4443.

[8] J. Elliott, L. Chan and T. Siu, "On Pricing Barrier Options with Regime Switching," Journal of Computational and Applied Mathematics, 2014, 256(1), pp. 196-210.

[9] N. Ratanov, "Option Pricing under Jump-Diffusion Processes with Regime Switching," Methodology and Computing in Applied Probability, 2016, 18, pp. 829-845.

[10] J. Ma and Z. Zhou, "Moving Mesh Methods for Pricing Asian Options with Regime Switching," Journal of Computational and Applied Mathematics, 2016, 298, pp. 211-221.

[11] X. Mao, "Stochastic Differential Equations and Applications," Horwood Publishing, Chichester, 2007.

[12] X. Mao and C. Yuan, "Stochastic Differential Equations with Markovian Switching,” Imperial College Press, 2006.

[13] C. Yuan and W. Glover, "Approximate Solutions of Stochastic Differential Delay Equations with Markovian Switching," Journal of Computational and Applied Mathematics, 2006, 194, pp. 207-226.

[14] K. Fan, Y. Shen, T. Siu and R. Wang, "On a Markov Chain Approximation Method for Option Pricing with Regime Switching," Journal of Industrial and Management Optimization, 2016, 12, pp. 529-541.

[15] Z. Jin and L. Qian, "Lookback Option Pricing for Regime Switching Jump Diffusion Models," Mathematical Control and Related Fields, 2015, 5, pp. 237-258.

[16] R. Merton, "The Theory of Rational Option Pricing," Bell Journal of Economics and Management Science, 1971, 4(4), pp. 141-183. 